\title{
ACÚMULO E CARACTERIZAÇÃO DA SERAPILHEIRA E FERTILIDADE DO SOLO EM COBERTURAS FLORESTAIS NO NORTE FLUMINENSE
}

\author{
Mirian Peixoto Soares da Silva', Antonio Carlos da Gama-Rodrigues ${ }^{2}$ \\ e Deborah Guerra Barroso
}

\section{RESUMO}

Os objetivos do estudo foram avaliar diversas coberturas florestais da Regiāo Norte Fluminense, relacionando o acúmulo de serapilheira, sua composição quimica e orgânica e a sua influência na melhoria da fertilidade do solo. Além de verificar o efeito de diluição nas amostragens de $0-5$ e $0-10 \mathrm{~cm}$ para avaliação dos indicadores. Foram avaliadas quatro áreas degradadas: duas destas, revegetadas com espécies leguminosas arbóreas (Acacia auriculiformis - acácia e a outra com Mimosa caesalpiniifolia - sabiá), uma pastagem e uma área em sucessāo secundária (capoeira). Em todas as coberturas foram coletadas amostras de solo nas profundidades de 0-5 e 0-10 cm para caracterização química, e amostras de serapilheira para caracterização quimica e orgânica (exceto para pastagem). Os componentes da serapilheira foram separados em folhas, galhos, estrutura reprodutiva e material fragmentado.

O acúmulo de serapilheira foi maior na cobertura de acácia, seguida pela capoeira e pelo sabiá. O maior acúmulo na cobertura de acácia pode estar relacionado a sua maior capacidade de produzir serapilheira ou que a decomposição e, ou, a renovação desta serapilheira seja mais lenta que as demais coberturas. Isso estaria relacionado à qualidade (orgânica e, ou quimica) do material produzido, determinando sua menor degradabilidade.

Para os atributos analisados, exceto $\mathrm{Ca}$, a acácia foi a cobertura que apresentou os menores teores nutricionais e as maiores relaçōes de Lignina/ $\mathrm{N}$ e Polifenóis $/ \mathrm{N}$, mostrando ser um material mais recalcitrante quando comparado com às demais coberturas. A análise quimica do solo sob as coberturas plantadas mostrou que a serapilheira influenciou na melhoria da fertilidade do solo, principalmente, na profundidade de $0-5 \mathrm{~cm}$.

Palavras chave: Ciclagem de nutrientes, recuperação de áreas degradadas, leguminosas arbóreas.

\footnotetext{
'Eng. Agrônoma. LFIT/CCTA/UENF. Av. Alberto Lamego, 2000 - Horto, 28013-602 Campos-RJ-Brasil. mirianpsoares@ig.com.br:

'Prof. LSOL/CCTA/UENF, tonygama@uenf.br;
} 


\section{ACCUMULATION AND CHARACTERIZATION OF THE LITTER FALL AND SOIL FERTILITY IN FOREST COVERINGS IN THE NORTH OF RIO DE JANEIRO STATE}

\section{SUMMARY}

The objective of the study was evaluating diverse forest covers in the North of Rio de Janeiro's State, Brazil, according to litter accumulation and its influence on soil fertility improvement. Four degraded areas are evaluated; two of these replanted with leguminous tree species (Acacia auriculiformis, acácia and Mimosa caesalpiniifolia, sabiá), a pasture and an area in secondary succession (naturally regenerated forest). Under each cover soil samples were collected in 0-5 and $0-10 \mathrm{~cm}$ depth per chemical characterization, and also litter samples per chemical and organic characterization (except for pasture). The components of the litter were separated in leaves, branches, structure and fragmented material.

The litter accumulation was bigger under Acacia cover, followed by the secondary succession and sabiá. The biggest accumulation under Acacia can be related to its better capacity to produce litter or may be the decomposition and renewal of this litter is slower than the other coverings. That would be related to a lower degradability of the produced material because of its organic or chemical quality.

Regarding to the analyzed attributes, except $\mathrm{Ca}$, Acacia was the cover with smaller nutritional tenors and the biggest relations of Lignin/ $\mathrm{N}$ and Polifenol/N, showing to be a more recalcitrant material when compared with the other covers. Soil chemical analysis under the planted covers showed that litter influenced the improvement of the soil fertility, mainly in the depth of $0-5 \mathrm{~cm}$.

Keywords: Nutrient cycling, recovery of degraded areas, leguminous trees. 


\section{CARACTERIZACIÓN DE LA ACUMULACIÓN DE LITTER Y LA FERTILIDAD DEL SUELO BAJO DISTINTAS CUBIERTAS FORESTALES EN EL NORTE DEL ESTADO DE RIO JANEIRO}

\section{RESUMEN}

El objetivo de este estudio es la evaluación de la acumulación de materia orgánica en el suelo bajo distintas cubiertas forestales y su influencia en el mejoramiento de la fertilidad de éste. Se evaluó cuatro áreas degradadas, dos de ellas plantadas con especies leguminosas arbóreas (Acacia auriculiformis, acacia, y Mimosa caesalpiniifolia, sabiá), una de pasturas y una con regeneración natural nativa. Bajo cada una de estas cubiertas se colectó muestras de suelo a profundidades de 0 - 5 y $0-10 \mathrm{~cm}$, para caracterización química, y muestras de litter para caracterización quimica y orgánica (excepto en el área de pasturas). Los componentes del litter fueron separados en hojas, ramas, estructura reproductiva y material fragmentado.

La acumulación de litter fue mayor bajo cobertura de acacia, seguida de la de regeneración natural y la de sabiá. La mayor acumulación bajo cubierta de acacia puede estar relacionada con su mayor capacidad de producir litter o deberse a que la descomposición de este material sea más lenta que bajo las otras cubiertas. Esto último podria estar relacionado con que la calidad quimica y orgánica del material producido determina una menor degradación.

Para los atributos analizados, con la excepción del $\mathrm{Ca}$, la acacia fue la cubierta que presentón los menores tenores nutricionales y las mayores relaciones lignina/ $\mathrm{N}$ y polifenol/ $\mathrm{N}$, mostrando ser un material más recalcitrante comparado con los de las otras cubiertas. El análisis quimico del suelo bajo las cubiertas plantadas mostró que el litter incidió el el mejoramiento de la fertilidad del suelo, principalmente en la profundidad $0-5 \mathrm{~cm}$.

Palabras claves: Ciclo de nutrientes, recuperación de suelos, leguminosas arbóreas. 


\section{INTRODUÇÃO}

Desde a colonização, o homem retira da natureza o seu sustento sem se preocupar com os possíveis danos ao ambiente. Com a Revolução Industrial, metade do Século XVIII, o processo de degradação foi acelerado, transformando radicalmente a vida tanto no campo como na cidade.

Desde então, a sustentabilidade dos ecossistemas vêm sendo comprometida pela contaminação do solo, pelo uso indiscriminado de agroquimicos, desmatamentos abusivos, queimadas, monocultivos, entre outros fatores. A falta de informação a respeito das conseqüências destes manejos adotados tem contribuido para intensificar ainda mais o processo de degradação.

Uma alternativa para minimizar esses efeitos é a manutenção ou implantação das coberturas florestais, que favorecem a deposição e o acúmulo de serapilheira no solo. Esta camada orgânica tem sido considerada o principal agente responsável pela ciclagem de nutrientes em ecossistemas florestais tropicais, mantendo a funcionalidade do sistema soloplanta, contribuindo positivamente para a reabilitação de áreas degradadas. A formação e a manutenção dessa camada garantem um reservatório de nutrientes, evitando que sejam perdidos por lixiviação ou erosão, sendo mineralizados lentamente (Andrade et al., 2000).

Vários fatores bióticos e abióticos interferem na produção de serapilheira, como: tipo de vegetação, altitude, latitude, precipitação, temperatura, luminosidade, relevo, deciduosidade, estágio sucessional, disponibilidade hídrica e características do solo (Figueiredo Filho, 2003).

O tempo em que os nutrientes permanecem estocados na serapilheira depende da velocidade de decomposição do material (Cole, 1981, citado por Monteiro, 2001). Coberturas florestais onde esteja presente um misto de espécies conferem à serapilheira uma diversidade de resíduos e esta diversidade pode contribuir no aumento da velocidade de decomposição, permitindo a atuação de diferentes grupos de macro e microrganismos decompositores.

O uso de espécies leguminosas arbóreas se destaca na revegetação de áreas degradadas pela capacidade da maioria delas formar simbiose com bactérias como Rhizobium, realizando a fixação biológica de nitrogênio (FBN), proporcionando um aporte de residuos que favorecem a ação de microrganismos decompositores. Entretanto há grande variação entre os residuos aportados pelas inúmeras espécies florestais tropicais.

A influência destes fatores na melhoria da fertilidade do solo por meio da decomposição da serapilheira é um processo lento e gradativo, podendo ser necessária a amostragem mais superficial para detecta-la no curto prazo. Não só a serapilheira, mas também a concentração de raízes finas nesta camada, está correlacionada com concentrações mais altas de matéria orgânica e nutrientes, e com as condiçōes físicas favoráveis desta camada (Witschoreck et al., 2003). Testar amostragens em diferentes profundidades podem auxiliar a avaliação desses indicadores. 


\section{OBJETIVOS}

Avaliar a qualidade de diversas coberturas florestais da Região Norte Fluminense, relacionando o acúmulo de serapilheira, sua composição quimica e orgânica e a sua influência na melhoria da fertilidade do solo, bem como o efeito de diluição nas amostragens $0-5$ e 0 $10 \mathrm{~cm}$ do solo.

\section{MATERIAIS E MÉTODOS}

O presente trabalho foi realizado a partir de amostras de solo e serapilheira coletadas na Fazenda Carrapeta, no Município de Conceiçāo de Macabu, RJ.

Neste local, predomina o relevo forte ondulado, com declividade em torno de $35 \%$. 0 solo é classificado como Latossolo Vermelho Amarelo, de textura argilo-franco-arenosa. $\mathrm{O}$ clima da região, pela classificação de Köppen, é do tipo Am, quente e úmido.

Foram avaliadas quatro diferentes áreas degradadas; duas destas revegetadas com espécies de leguminosas arbóreas (uma com a Acacia auriculiformis, acácia, e a outra com Mimosa caesalpiniifolia, sabiá); uma pastagem com predomínio do capim sapê, ao lado das áreas revegetadas; e uma área formada por um fragmento florestal da Mata Atlântica em sucessão secundária, capoeira, ao lado da pastagem. As duas últimas áreas citadas são utilizadas como referência.

Em setembro 2003 (plantios com 4 anos e nove meses de idade) foram coletadas em cada cobertura vegetal oito amostras compostas de solo para caracterização quimica, sendo quatro na profundidade de $0-10 \mathrm{~cm}$ (obedecendo à amostragem ao acaso de quinze amostras simples por amostra composta), e quatro na profundidade de $0-5 \mathrm{~cm}$ (oito amostras simples, ao acaso, por amostra composta). No caso das áreas revegetadas, a coleta foi feita entre linhas de plantio. Para a coleta a $0-10 \mathrm{~cm}$, foi utilizado um trado holandês. As amostras de solo, das duas profundidades, foram secas ao ar. Após a secagem, as amostras foram devidamente passadas em peneira de malha $2 \mathrm{~mm}$.

$\mathrm{Na}$ área do pasto não ocorre acúmulo de serapilheira. Para as demais áreas, no entanto, a amostragem da serapilheira, para caracterização quimica e orgânica, foi realizada utilizando-se um gabarito (quadrado de madeira) de $0,25 \mathrm{~m}^{2}$. Nas áreas plantadas com acácia, e com sabiá foram coletadas seis amostras simples, enquanto na capoeira foram coletadas quatro amostras simples. As amostras foram coletadas ao acaso e, no caso das áreas revegetadas, entre linhas de plantio. Os componentes da serapilheira foram devidamente separados (folha, galho, estrutura reprodutiva e material fragmentado) e submetidos à secagem em estufa, a $75^{\circ} \mathrm{C}$, até peso constante. Para que fosse reduzido o problema de contaminação pelo solo na análise da serapilheira, a separação desses materiais foi feita sendo descartado todo material de solo encontrado. As amostras de serapilheira foram pesadas em balança de precisão de $0,1 \mathrm{~g}$ e posteriormente moidas para análise.

Nas amostras de solo foram realizadas as determinações de $\mathrm{P}$ e $\mathrm{K}$ (extraiveis por Mehlich-1), Ca, Mg e Al (trocáveis, por KCl 1 mol/L), (Defelipo e Ribeiro, 1981), C orgânico 
por oxidação com $\mathrm{K}_{2} \mathrm{Cr}_{2} \mathrm{O}, 1,25 \mathrm{~mol}_{c} / \mathrm{L}$ em meio ácido (Anderson e Ingram, 1996), $\mathrm{N}$ total pelo método Kjeldahl, conforme o método descrito pela EMBRAPA (1997) e pH (emágua).

Determinaram-se, nos diferentes componentes da serapilheira, os teores de $\mathrm{K}$ (fotometria de chama), de $\mathrm{P}$ (colorimetricamente pelo método da vitamina $\mathrm{C}$, modificado por Braga e Defelipo, 1974), de Ca e Mg (espectrofotometria de absorção atômica), após digestão nitro-perclórica, e de $\mathrm{N}$ pelo método Kjedahl, descrito por Bataglia et al. (1983). O teor de C foi obtido por oxidação com $\mathrm{K}_{2} \mathrm{Cr}_{2} \mathrm{O}_{7}$ (Anderson e Ingram, 1996). Na determinação de lignina e celulose da serapilheira foi empregado o método da fibra em detergente ácido (FDA) de van Soest e Wine (1968) e para polifenóis solúveis totais, o método Folin-Denis, descrito por Anderson e Ingram (1996).

Para a análise estatistica dos dados considerou-se um delineamento experimental inteiramente casualizado, sendo aplicado, para a comparação das médias, o teste de Tukey a $5 \%$ de probabilidade. Utilizou-se o programa estatistico SAEG (Funarbe, 1993) para o procedimento das análises.

\section{RESULTADOS E DISCUSSÃO}

O acúmulo de serapilheira foi maior no povoamento da acácia $\left(1058,04 \mathrm{~g} / \mathrm{m}^{2}\right)$, sendo superior ao acúmulo do sabiá $\left(667,4 \mathrm{~g} / \mathrm{m}^{2}\right)$. A quantidade de serapilheira acumulada pela capoeira foi intermediária $\left(729,1 \mathrm{~g} / \mathrm{m}^{2}\right)$, não diferindo estatisticamente das demais coberturas (Tabela 1). Esses resultados estão na mesma faixa dos encontrados por Gama-Rodrigues et al. (1999), onde trabalhando com seis espécies nativas plantadas em parcelas puras (pauroxo, putumuju, arapati, arapaçu, claraíba e ópleo-comumbá), com 22 anos de idade; em plantio misto (essas seis espécies associadas com outras 57 espécies nativas e exóticas); uma floresta secundária, praticamente em estado climax; e uma capoeira de 40 anos de idade, encontraram para os ecossistemas heterogêneos valores de fitomassa de serapilheira intermediários, em comparação com os plantios puros.

Provavelmente, este maior acúmulo de serapilheira apresentado pela acácia pode estar relacionado a sua maior capacidade de produzir serapilheira ou que a decomposição e, ou, a renovação desta serapilheira seja mais lenta que as demais coberturas. Isso estaria relacionado à qualidade (orgânica e, ou química) do material produzido, determinando sua menor degradabilidade (Tabelas 2 e 3). Mesma tendência foi encontrada por Andrade et al. (2000), trabalhando com povoamentos homogêneos de Mimosa caesalpiniifolia, Acacia mangium e Acacia holosericea, com aproximadamente quatro anos de idade.

Zaia e Gama-Rodrigues (2004), estudando a ciclagem de nutrientes em povoamentos de Eucalyptus grandis, E. camaldulensis e E. pellita, com 6 anos de idade, na Região Norte Fluminense, encontraram menor acúmulo de serapilheira para Eucalyptus grandis e concluiram que este resultado poderia estar relacionado a características do material como baixa relação $\mathrm{C} / \mathrm{N}$, menores teores de lignina, polifenóis e celulose, já que não foi encontrada grande diferença nutricional da serapilheira analisada. 
$\mathrm{Na}$ acácia e na capoeira, a folha foi a estrutura que mais contribuiu para a acúmulo de serapilheira, enquanto, no sabiá, foi o material fragmentado (Tabela 1).

\section{Tabela 1 \\ ACUMULAÇÃO DE MATÉRIA SECA DOS COMPONENTES DA ACACIA, SABIÁ E CAPOEIRA, COM 57 MESES DE IDADE, CULTIVADOS EM CONCEIÇĀO DE MACABU, RJ.}

\begin{tabular}{|c|c|c|c|c|c|}
\hline Cobertura & Folha & Galho & $\begin{array}{c}\text { Estrutura } \\
\text { Reprodutuva }\end{array}$ & $\begin{array}{c}\text { Material } \\
\text { Fragmentado }\end{array}$ & Total \\
\cline { 2 - 6 } & \multicolumn{3}{|c|}{$\left(\mathbf{g}^{\mathbf{2}} \mathbf{)}\right)$} & $236,3 \mathrm{a}$ & $1058,0 \mathrm{a}$ \\
Acácia & $579,6 \mathrm{a}$ & $15,8 \mathrm{~b}$ & 226,3 & $309,8 \mathrm{a}$ & $667,4 \mathrm{~b}$ \\
Sabia & $269,2 \mathrm{~b}$ & $88,4 \mathrm{a}$ & - & $222,4 \mathrm{a}$ & $729,1 \mathrm{ab}$ \\
Capoeira & $379,2 \mathrm{ab}$ & $127,5 \mathrm{a}$ & - & 30,07 & 27,0 \\
\hline
\end{tabular}

Médias seguidas de pelo menos uma mesma letra em cada coluna, nâo diferem entre si pelo teste de Tukey a $5 \%$ de probabilidade, $\mathrm{CV}$ : coeficrente de variaçăo.

Diversos fatores estão relacionados com a decomposição dos resíduos vegetais adicionados ao solo, tais como características edafoclimáticas, composição quimica dos residuos e estratégias de manejo. Sob as mesmas condições de clima e solo, a velocidade de decomposição dos resíduos e a liberação de nutrientes são afetadas por características químicas dos resíduos. Materiais com baixa relação $\mathrm{C} / \mathrm{N}(<25)$ e reduzidos teores de lignina e polifenóis apresentam mineralização rápida e fornecem grandes quantidades de nutrientes para as culturas subseqüentes. Já os materiais com elevada relação $\mathrm{C} / \mathrm{N}(>25)$ e altos teores de lignina e polifenóis sofrem decomposição mais lenta, podendo formar uma cobertura morta estável e capaz de proteger o solo contra a erosão (Myers et al., 1994 citado por Ndaw, 2003).

Contudo, os valores isolados de lignina, celulose e polifenóis, normalmente, não são bons indicadores desta decomposição (Gama-Rodrigues et al., 1999), ou seja, podem não explicar a maior ou menor acumulação de serapilheira. Por isso, devem ser feitas relações com o N, por se tratar de um nutriente limitante à atividade microbiana (Tabela 3). Em sistemas cujas relações apresentam valores mais elevados, a decomposição e a liberação de $\mathrm{N}$ são mais baixas (Gama-Rodrigues, 2004).

Os resultados da análise química da folha para os teores de $C_{\text {, lignina e polifenóis }}$ não apresentaram diferença significativa entre as coberturas vegetais. Para $\mathrm{N}$ e $\mathrm{P}$ os teores encontrados na acácia foram estatisticamente inferiores em relação às demais coberturas (Tabelas 2 e 3). Para os teores de Mg e celulose, os resultados nas folhas da serapilheira de capoeira foram estatisticamente superiores em relação às outras coberturas estudadas. Nas folhas de capoeira foi observado maior teor de $\mathrm{Ke}$ menor teor de $\mathrm{Ca}$ em relação às demais áreas (Tabelas 2 e 3). Monteiro e Gama-Rodrigues (2004), trabalhando com diferentes 
estruturas de serapilheira de uma floresta natural, observaram, para os teores de $\mathrm{C} \mathrm{e} \mathrm{N}$ analisados da folha, valores similares dos encontrados neste estudo. Sob capoeira foi observado o maior teor de $\mathrm{K}$ nas folhas, entretanto foi a cobertura que apresentou o menor teor de $\mathrm{Ca}$ entre todos os componentes da serapilheira analisados. Na serapilheira de acácia foi observada a maior relação $\mathrm{C} / \mathrm{N}$ diferindo estatisticamente das demais coberturas.

Tabela 2

CONCENTRAÇÃO MÉDIA DE NUTRIENTES NOS COMPONENTES DA SERAPILHEIRA DAS DIFERENTES COBERTURAS VEGETAIS

\begin{tabular}{|c|c|c|c|c|c|c|c|}
\hline \multirow[t]{2}{*}{ Cobertura } & C & $\mathbf{N}$ & $\mathbf{P}$ & $\bar{K}$ & Ca & $\mathbf{M g}$ & CN \\
\hline & \multicolumn{7}{|c|}{ (Ekg) } \\
\hline & \multicolumn{7}{|c|}{ Folin } \\
\hline Acticin & $360,5 a$ & 15,10 & $0,19 \mathrm{~b}$ & $0,73 \mathrm{~b}$ & $22.39 a$ & 1940 & $23,87 a$ \\
\hline Satud & $362,5 a$ & $17,9 \mathbf{a}$ & $0,43 a$ & $0.93 a b$ & $1749 a$ & 2110 & 20.250 \\
\hline Capoelra & $350.2 \mathrm{a}$ & $18,4 a$ & $0.41 \mathrm{a}$ & $1,30 a$ & $815 b$ & 3350 & 19036 \\
\hline \multirow[t]{2}{*}{ C.V. $\%$} & 5,21 & 5.93 & 13,26 & 23,11 & 2502 & 24,79 & 7.87 \\
\hline & \multicolumn{7}{|c|}{ Oalliso } \\
\hline Acticle & $3619 b$ & $120 \mathrm{a}$ & $0,31 \mathbf{a}$ & $093 a$ & $25,47 a$ & $1,13 \mathrm{~b}$ & $30,16 a$ \\
\hline Sabuth & $386,1 \mathrm{a}$ & $13,1 \mathbf{a}$ & $0,33 \mathbf{a}$ & $0.90 a$ & $1087 b$ & $2,01 a$ & $29,47 \mathrm{a}$ \\
\hline Cepoetre & 356,10 & $12,8 \mathrm{a}$ & $0.33 \mathbf{a}$ & $100 a$ & $8.88 \mathrm{~b}$ & $207 a$ & $2760 a$ \\
\hline c.v. $\%$ & 3,38 & 10,59 & 1896 & 30.26 & 36,99 & 2861 & 1039 \\
\hline \multirow[b]{2}{*}{ Actcia } & \multicolumn{7}{|c|}{ Estrufure Roprodutiva } \\
\hline & 378,4 & 12.5 & 0,20 & 1.00 & 5,23 & 069 & 3027 \\
\hline & \multicolumn{7}{|c|}{ Meteriel Fregmentado } \\
\hline Actacla & $3256 a$ & $16.6 a$ & $0,34 \mathrm{~b}$ & $0.80 \mathrm{~b}$ & $1593 a$ & $1,61 \mathrm{~b}$ & $19.61 \mathrm{a}$ \\
\hline Sabid & $324,6 a$ & $18,6 \mathbf{a}$ & $0,51 \mathbf{a}$ & $1,03 \mathrm{ab}$ & $17,45 a$ & $2,33 b$ & $17,45 a$ \\
\hline Capoetre & $321,3 a$ & $19,4 a$ & $0,55 \mathbf{a}$ & $1,10 \mathrm{a}$ & $10.48 a$ & $3,42 \mathrm{a}$ & $1656 a$ \\
\hline c.v. $\%$ & 8,26 & 17,74 & 13,98 & 16,60 & 32,11 & 25,45 & 19.84 \\
\hline
\end{tabular}

Medias seguidas de pelo menos uma mesma letra em cada componente nao dilerem entre si pelo teste de Tulvey a $5 \%$ de probabilidade, CV

Coeficente de vanaço

Tabela 3

TEORES DE POLIFENOIS (POL), LIGNINA (LIG) E CELULOSE (CEL) E SUAS RESPECTIVAS RELAÇOES COM NITROGÊNIO DA SERAPILHEIRA NAS DIFERENTES COBERTURAS VEGETAIS ESTUDADAS

\begin{tabular}{|c|c|c|c|c|c|c|}
\hline \multirow[t]{2}{*}{ Cobertum } & POL & 10 & cal & LON & (10+C्वप्याN & POLN \\
\hline & \multicolumn{6}{|c|}{ (olve) } \\
\hline & \multicolumn{6}{|c|}{ Folitie } \\
\hline Actela & 9,60 & $452.70 \mathrm{a}$ & $19270 \mathrm{~b}$ & $30,00 a$ & $42,74 a$ & $0.64 \mathrm{a}$ \\
\hline Sabu & $9.60 \mathrm{a}$ & $478,00 \mathrm{a}$ & $214.70 \mathrm{~b}$ & $26,70 \mathrm{~b}$ & $38.70 \mathrm{a}$ & $0,54 \mathrm{ab}$ \\
\hline Capoeira & $7,70 \mathrm{a}$ & $477,00 \mathrm{a}$ & $32300 \mathrm{a}$ & $25.92 \mathrm{~b}$ & $43.48 \mathrm{a}$ & $0.42 \mathrm{~b}$ \\
\hline \multirow[t]{2}{*}{ c.v.\% } & 19,69 & 6.08 & 9.75 & 7,53 & 7,37 & 22,42 \\
\hline & \multicolumn{6}{|c|}{ Galho } \\
\hline Acicia & $6,70 \mathrm{a}$ & $324.00 \mathrm{~b}$ & $386,00 \mathrm{a}$ & $27,00 \mathrm{a}$ & $59.17 \mathrm{a}$ & $0,56 \mathbf{a}$ \\
\hline Sabu & $6,70 \mathrm{a}$ & $414.70 a$ & $334,70 \mathrm{~b}$ & $31,66 a$ & $5721 a$ & $0,51 \mathrm{a}$ \\
\hline Capoetre & $4,10 \mathrm{a}$ & $361,00 \mathrm{~b}$ & $374,00 \mathrm{ab}$ & $27.98 \mathrm{a}$ & $56.98 a$ & $0.32 \mathrm{a}$ \\
\hline C.V.\% & 2586 & 7.06 & 6.98 & 13.65 & 1194 & 25,65 \\
\hline \multirow[b]{2}{*}{ Actele } & \multicolumn{6}{|c|}{ Estrutur Reproditiva } \\
\hline & 128 & 32800 & 347.30 & 26.24 & 54,02 & 1.02 \\
\hline & \multicolumn{6}{|c|}{ Mattertal Fregonortado } \\
\hline Acticia & $3,30 \mathrm{a}$ & $450.7 \mathrm{~b}$ & $195,30 \mathrm{a}$ & $27,15 a$ & $3892 a$ & $0,20 \mathrm{a}$ \\
\hline Sabu & $3,10 \mathrm{a}$ & $568.0 a$ & $170,70 a$ & $30,54 a$ & $39,71 \mathbf{a}$ & $0.17 \mathbf{a}$ \\
\hline Capoenin & $3,80 \mathrm{a}$ & $521,0 \mathrm{ab}$ & $218.00 \mathrm{a}$ & $26.86 a$ & $38,09 a$ & $0.20 \mathrm{a}$ \\
\hline c.v.\% & 25,20 & 8.07 & 20,00 & 1685 & 18,47 & 26,29 \\
\hline
\end{tabular}

Medias seguidas de pelo menos uma mesma letra em cada componente nao diferem entre $s$ pelo teste de Tuley a $5 \%$ de probabilidade CV Coetcente de Variacao 
Os resultados da análise química dos galhos da serapilheira para os teores de N, P, $\mathrm{K}, \mathrm{C} / \mathrm{N}$ e polifenóis foram estatisticamente iguais entre as coberturas. Já para os demais componentes, foram encontradas diferenças significativas: maiores teores de $\mathrm{C}$ e lignina foram observados sob sabiá e menor teor de $\mathrm{Mg}$ sob acácia, onde também foram encontrados os maiores valores de $\mathrm{Ca}$ e celulose (Tabelas 2 e 3 ). A estrutura reprodutiva estava presente na serapilheira apenas sob plantio de acácia na época da amostragem.

Quanto aos resultados do material fragmentado, os teores de $\mathrm{C}, \mathrm{N}, \mathrm{Ca}, \mathrm{C} / \mathrm{N}$, celulose e polifenóis foram similares entre as diferentes coberturas. Neste componente, os menores teores de P, K, Mg e lignina foram verificados na acácia (Tabelas 2 e 3). Em geral, o $\mathrm{K}$ é o nutriente de mais rápida liberação da serapilheira em todos os ecossistemas (GamaRodrigues, 1997). Malavolta (1980) relatou que $80 \%$ do K do tecido seria passivel de lavagem, pelo fato de tal elemento encontrar-se quase que totalmente na forma solúvel. Os resultados encontrados por Roselem'et al. (2003) mostram que essa lavagem depende também de outros fatores, além da chuva. Provavelmente, a maior liberação do $\mathrm{K}$ depende também da degradação dos restos vegetais. A degradação biológica deste material implicaria no rompimento das barreiras à difusão do K, facilitando sua liberação. Isto pode ter um importante significado ecológico na reciclagem de nutrientes e na fertilização das camadas superficiais do solo.

Para a relação LIG/N nas folhas, a acácia foi a cobertura que apresentou o maior valor, sendo estatisticamente superior as demais coberturas neste componente analisado. Já para o galho e o material fragmentado, não houve diferença desta relação entre as coberturas analisadas (Tabela 3 ).

Para a relação (LIG + CEL)/N, em todos os componentes analisados não houve diferença estatística entre as coberturas (Tabela 3 ).

Para a relação POL/N, na folha, a capoeira apresentou o menor valor. Nos componentes galho e material fragmentado, não houve diferença estatistica entre as coberturas analisadas (Tabela 3 ).

Para os atributos analisados, exceto $\mathrm{Ca}$, a acácia foi a cobertura que apresentou os menores teores nutricionais e as maiores de LIG/N e POL/N, mostrando ser um material mais recalcitrante quando comparado com às demais coberturas (Tabelas 2 e 3 ).

O aporte de nutrientes de cada cobertura é influenciado não apenas pela característica nutricional das fraçōes da serapilheira acumulada, mas principalmente por sua relação com as quantidades produzidas, refletindo na proporção de nutrientes transferida através dessa via.

A folha foi o componente que acumulou a maior quantidade de nutrientes em todas as coberturas analisadas (Tabela 4).

Os resultados da análise quimica para $\mathrm{C}$ e $\mathrm{N}$ do solo, sob as diferentes coberturas vegetais, nas duas profundidades, não apresentaram diferença significativa (Tabela 5). 
Entretanto, pode-se afirmar que houve um incremento do $C$ orgânico, pois ao analisar as amostras, aos 57 meses de idade, foram encontrados teores médios de $26,7 \mathrm{~g} / \mathrm{kg}$, em amostragem realizada de $0-10 \mathrm{~cm}$, enquanto que Paulino (2003) encontrou valores abaixo de $17 \mathrm{~g} / \mathrm{kg}$, aos 32 meses de idade na mesma área de plantio, com a mesma amostragem. $\mathrm{O}$ autor relacionou este resultado ao fato de que não havia sido formada serapilheira, como reflexo da curta idade dos cultivos. Com a amostragem realizada de $0-5 \mathrm{~cm}$ foi encontrada variação na faixa de 29,4 a $32 \mathrm{~g} / \mathrm{kg}$ e Gama-Rodrigues et al. (1997), avaliando um Latossolo Vermelho Amarelo argiloso sob angico, pinheiro e eucalipto, todos com 25 anos de idade, também não encontraram variação no teor de $C$ orgânico, apesar da quantidade de serapilheira acumulada entre as coberturas ter variado. $O$ incremento do $C$ no solo é reflexo da decomposição da serapilheira, mas também das raizes que contribuem bastante para melhoria da fertilidade, principalmente na camada mais superficial do solo.

A acidez ativa do solo foi considerada elevada em todas as coberturas e profundidades estudadas. O solo sob a capoeira apresentou o valor de $\mathrm{pH}$ mais baixo, nas duas profundidades. Também os maiores teores de Al trocável foram encontrados no solo sob a capoeira. Este resultado pode ser decorrente desse baixo $\mathrm{pH}$ verificado, resultando na mais elevada acidez potencial $(\mathrm{H}+\mathrm{Al})$. Em ambas as amostragens, os teores de alumínio trocável encontrados para acácia, sabiá e pasto não diferiram significativamente entre si.

Tabela 4

CONTEỦDO MÉDIO DE NUTRIENTES NOS COMPONENTES DA SERAPILHEIRA DAS DIFERENTES COBERTURAS VEGETAIS

\begin{tabular}{|c|c|c|c|c|c|c|}
\hline \multirow[t]{2}{*}{ Cobertura } & C & $\mathbf{N}$ & $\mathbf{P}$ & $\mathbf{K}$ & Ca & Mg \\
\hline & \multicolumn{6}{|c|}{ (kgha) } \\
\hline & \multicolumn{6}{|c|}{ Folha } \\
\hline Acácia & 208382 a & $87.83 \mathbf{a}$ & $1.09 a$ & $4.24 \mathrm{a}$ & $132.91 \mathrm{a}$ & $11,14 \mathrm{a}$ \\
\hline Sabda & $979.33 \mathrm{~b}$ & $4791 \mathrm{~b}$ & $1,17 \mathrm{a}$ & 256 a & $4945 b$ & $576 \mathrm{~b}$ \\
\hline \multirow[t]{2}{*}{ Capoeira } & $1310,96 \mathrm{~b}$ & $6934 \mathrm{ab}$ & $1,56 \mathrm{a}$ & $488 a$ & $2980 \mathrm{~b}$ & $12.35 \mathrm{a}$ \\
\hline & \multicolumn{6}{|c|}{ Galho } \\
\hline Acacia & $58,37 \mathrm{~b}$ & $2,01 \mathrm{~b}$ & $005 \mathrm{~b}$ & $0,17 \mathrm{~b}$ & $4,41 \mathrm{a}$ & $0.20 \mathrm{~b}$ \\
\hline Sabea & $341.59 a$ & $11.55 \mathrm{a}$ & $0.29 a$ & $0.79 \mathrm{ab}$ & 9,81 a & $1,77 \mathrm{ab}$ \\
\hline \multirow[t]{2}{*}{ Capoeira } & 44861 a & $1623 a$ & $0,41 \mathbf{a}$ & $127 \mathbf{a}$ & $11.20 \mathrm{a}$ & $267 a$ \\
\hline & \multicolumn{6}{|c|}{ Estrutura Peprodutiva } \\
\hline \multirow[t]{2}{*}{ Acacta } & 85588 & 27.54 & 0.46 & 2.20 & 12.35 & 162 \\
\hline & \multicolumn{6}{|c|}{ Material Fragmentado } \\
\hline Acácia & $763,82 \mathrm{a}$ & 39.47 a & 0.820 & $1,89 \mathrm{~b}$ & 38,98 a & 393 a \\
\hline Sabla & 995,08 a & 58,07 a & $1,60 \mathbf{a}$ & $3,22 \mathbf{a}$ & $5660 a$ & $7,10 a$ \\
\hline Capoeira & $71680 \mathrm{a}$ & 43.65 a & $123 \mathrm{ab}$ & $2,43 \mathrm{ab}$ & $23,29 \mathrm{a}$ & $7,55 \mathrm{a}$ \\
\hline
\end{tabular}

Médias seguidas de pelo menos uma mesma letra em cada componente nâ diferem entre si pelo teste de Tukey a $5 \%$ de probabilidade, $\mathrm{CV}$ probablidade 
Os maiores teores de $\mathrm{Ca}, \mathrm{Mg}$ e $\mathrm{K}$ na amostragem de $0-5 \mathrm{~cm}$ foram observados nos solos sob acácia e sabiá, enquanto o solo sob capoeira apresentou os menores valores, com valores médios de 0,$73 ; 0,68$ e $0,13 \mathrm{cmolc} / \mathrm{dm}^{3}$, respectivamente. Não houve diferença entre os teores de $\mathrm{K}$ observados para acácia, sabiá e pasto nas duas profundidades; entretanto, o K nessas coberturas foi superior à capoeira. $\mathrm{Na}$ amostragem de $0-5 \mathrm{~cm}$, o solo sob acácia apresentou maior teor de $\mathrm{P}$, diferindo significativamente das demais coberturas. Este resultado pode ser explicado pela mineralização deste nutriente da serapilheira de acácia. Os teores de P na amostragem de $0-10 \mathrm{~cm}$ não apresentaram diferença entre as coberturas vegetais estudadas e foram inferiores aos observados na amostragem de $0-5$ $\mathrm{cm}$, o que pode ser explicado pelo fato deste elemento apresentar baixa mobilidade no perfil do solo (Tabela 5), indicando um efeito de diluição em amostragem mais profunda.

Tabela 5

VALORES MÉDIOS DA ANÁLISE QUIMICA DO SOLO SOB DIFERENTES COBERTURAS VEGETAIS

\begin{tabular}{|c|c|c|c|c|c|c|c|c|c|c|}
\hline \multirow[t]{2}{*}{ Cobertura } & \multirow{2}{*}{$\begin{array}{c}\text { Profundidade } \\
\text { (cm) }\end{array}$} & C & $\mathbf{N}$ & \multirow[t]{2}{*}{ pH } & $\mathbf{P}$ & $\mathbf{K}$ & $\mathrm{Ca}$ & $M g$ & $\mathbf{A}$ & $\mathbf{H}+\mathbf{A}$ \\
\hline & & \multicolumn{2}{|c|}{ (g/kg) } & & \multicolumn{2}{|c|}{ (moldm ') } & \multicolumn{4}{|c|}{ (cmole, $\left(1 \mathrm{dm}^{3}\right)$} \\
\hline Actacia & 0.5 & 3190 & $1,50 \mathrm{a}$ & $508 a$ & $5.26 \mathrm{a}$ & $13600 \mathrm{~A}$ & $2.37 \mathrm{a}$ & 1,2000 & 0.180 & 3960 \\
\hline Sabia & 0.5 & 309 & $1,30 \mathrm{a}$ & $4.91 a$ & 400 & $13300 \mathrm{a}$ & $2,00 \mathrm{ab}$ & $1.25 a$ & 0,400 & $5.78 a b$ \\
\hline Pasto & 0.5 & $294 a$ & $1.20 \mathrm{~B}$ & $4.85 a$ & 3.880 & $105.00 \mathrm{a}$ & $1,47 b c$ & $0,82 x$ & 0.436 & $565 a b$ \\
\hline Capoeira & 0.5 & $31.2 a$ & $1,60 \mathrm{a}$ & 4.240 & 4050 & 50750 & $0.73 c$ & $068 \mathrm{C}$ & $098 a$ & $6.36 a$ \\
\hline CV $\%$ & 0.5 & 806 & 2806 & 369 & 8.24 & 19.4 & 24,16 & 1850 & 2879 & 1861 \\
\hline Sabid & 0.10 & 26.37 & 1,600 & $474 a$ & $329 a$ & $102,75 \mathrm{a}$ & $1.31 a b$ & $096 a$ & 0.630 & $5,+\infty 0$ \\
\hline Pasto & 0.10 & 26,80 & $130 \mathrm{a}$ & $475 a$ & $3.13 a$ & $108.00 \mathrm{a}$ & $1.42 \mathrm{a}$ & 0,8030 & 0600 & 5,080 \\
\hline Capoeira & 0.10 & $298 a$ & $160 \mathrm{a}$ & $4,12 b$ & $372 a$ & 47.250 & 0,550 & 0.490 & 1.400 & $7.22 \mathrm{a}$ \\
\hline CV $\%$ & 0.10 & $7 . \infty$ & 2133 & 347 & 1433 & 1226 & 3039 & 19,95 & 39,97 & 618 \\
\hline
\end{tabular}

Médes segudas de peo menos uma mesma letra em cada prdunddade náo dierem entre si peb teste de Tukey a $5 \%$ de probabidade CV Coetcerte de varaço

Portanto, ao comparar as características quimicas do solo sob as coberturas florestais plantadas com a capoeira e o pasto, verificou-se que houve alterações significativas na fertilidade, detectada principalmente na amostragem mais superficial $(0-5 \mathrm{~cm})$, apresentando maior sensibilidade para $\mathrm{Ca}$ e $\mathrm{Mg}$ e menor para acidez potencial, sendo o consórcio com essas espécies uma alternativa para minimizar os custos com fertilizantes quimicos.

Sugere-se a análise tanto do solo, quanto da serapilheira em diferentes estações do ano e em diferentes profundidades de solo, pois, desta forma, seria possivel obter informações a respeito de quais fatores climáticos desta região seriam mais limitantes para acumulação e decomposiçāo da serapilheira, bem como sua contribuição para melhoria das caracteristicas químicas, fisicas e biológicas do solo.

A amostragem de $0-5 \mathrm{~cm}$ mostrou-se mais sensivel na detecção de diferenças de alguns indicadores entre as coberturas analisadas, isto pode ser verificado ao observar os conteúdos de $\mathrm{P}, \mathrm{Ca}$ e $\mathrm{Mg}$ acumulados (Tabela 6). 
Tabela 6

CONTEÜDO DE NUTRIENTES NO SOLO EM DUAS AMOSTRAGENS, SOB DIFERENTES COBERTURAS VEGETAIS

\begin{tabular}{|c|c|c|c|c|c|c|c|}
\hline \multirow[t]{2}{*}{ Cobertura } & \multirow{2}{*}{$\begin{array}{l}\text { Profundidade } \\
\text { (cm) }\end{array}$} & C & $\mathbf{N}$ & $\mathbf{P}$ & $\mathbf{K}$ & $\mathrm{Ca}$ & $\mathbf{M g}$ \\
\hline & & \multicolumn{6}{|c|}{ (kg/ha) } \\
\hline Acácia & 0.5 & 7.97 a & 750,00 a & $2.63 \mathrm{a}$ & 68,00 a & 236,44 a & $72,88 \mathrm{ab}$ \\
\hline Sabiá & 0.5 & $7,73 \mathbf{a}$ & 650,00 a & $2,21 \mathrm{~b}$ & 66.50 a & $199,65 \mathrm{ab}$ & 76,33 a \\
\hline Pasto & 0.5 & $7.35 \mathbf{a}$ & $612.50 \mathrm{a}$ & $1,94 \mathrm{~b}$ & $52.50 \mathrm{a}$ & $146,60 \mathrm{bc}$ & $49,83 \mathrm{bc}$ \\
\hline Capoeira & 0.5 & $6,88 \mathrm{a}$ & $787,50 \mathrm{a}$ & $2,03 \mathrm{~b}$ & $25,38 \mathrm{~b}$ & $73,15 \mathrm{c}$ & $41,21 \mathrm{c}$ \\
\hline Acácia & 0.10 & 13,46 a & $1500,00 \mathrm{a}$ & $3.40 \mathrm{a}$ & 103,00 a & 358,02 a & $110.63 \mathrm{a}$ \\
\hline Sabiá & $0-10$ & 13,15 a & 1575,00 a & 3,29 a & $102,75 \mathrm{a}$ & $261,35 a b$ & $116,80 \mathrm{a}$ \\
\hline Pasto & 0.10 & $13,40 \mathrm{a}$ & $1266,67 a$ & $3,13 \mathrm{a}$ & $108,00 \mathrm{a}$ & 309.89 a & $97.39 a b$ \\
\hline Capoeira & $0-10$ & 14,91 a & $1600,00 \mathrm{a}$ & $3.72 \mathrm{a}$ & $47.25 \mathrm{~b}$ & $109,28 \mathrm{~b}$ & $58.94 \mathrm{~b}$ \\
\hline
\end{tabular}

Medias seguidas de pelo uma mesma letra em cada profundidade não diferem entre si pelo teste de Tukey a $5 \%$ de probabilidade. Profundidade $1: 0.5 \mathrm{~cm}$ e Profundidade 2: 0-10 cm;

Os resultados aqui apresentados ressaltam a importância da profundidade de amostragem, mostrando que a amostragem da camada mais superficial do solo è mais recomendada para verificação da contribuição da decomposição da serapilheira e de outros materiais, como as raizes e componentes da microfauna, no incremento de nutrientes no solo (Tabela 6).

\section{CONCLUSÕES}

O uso de leguminosas arbóreas apresenta um grande potencial na recuperação de áreas degradadas;

O plantio florestal, através da acumulação de serapilheira, altera positivamente a fertilidade do solo;

O acúmulo de serapilheira, em parte, está relacionado com a qualidade do seu material formador;

A amostragem de $0-5 \mathrm{~cm}$ de profundidade é mais representativa que de $0-10$ $\mathrm{cm}$ para detectar diferenças de $\mathrm{P}, \mathrm{Ca}$ e $\mathrm{Mg}$ no solo, resultantes do efeito de diferentes coberturas florestais. 


\section{REFERÊNCIAS}

Anderson, J. N.; Ingram, J. S. I., 1996. Tropical Soil Biology and Fertility: A Handbook of Methods. 1.ed. Wallingford, Cab International, UK, $171 \mathrm{p}$.

Andrade, A. G.; Costa, G. S. e Faria, S. M., 2000. Deposiçāo e Decomposição da Serapilheira em Povoamentos de Mimosa caesalpiniifolia, Acacia mangium e Acacia holosericea com quarto anos de idade em planossolo. R Bras. Ci. Solo, (24): 777-785.

Bataglia, O. C., Furlani, A. M. C., Teixeira, J. P. F., Furlani, P. R. e Gallo, J. R., 1983. Métodos de Análise Quimica de Plantas. Instituto Agronômico, Campinas, 48p. (Boletim Técnico, 78).

Braga,J.M. e Defelipo, B. V., 1974. Determinação Espectrofotométrica de Fósforo em Extrato de Solo e Material Vegetal. R. Ceres, Viçosa, (21): 73-85.

Defelipo, B. V. e Ribeiro, A. C., 1981. Análise Quimica do Solo. Viçosa, UFV, 17p. (Boletim de Extensão, 29).

EMBRAPA. Serviço Nacional de Levantamento e Conservação de Solos, 1997. Manual de Métodos de Análise de Solos. 2.ed. ver. atual. Rio de Janeiro, snlcs.

Ferreira, C. A. e Galvão, A. P. M., 2000. Importância da Atividade Florestal no Brasil. in: Galvão, A. P. M. (ed.) Reflorestamento de Propriedades Rurais para Fins Produtivos e Ambientais: Um Guia para Açōes Municipais e Regionais, EMBRAPA Comunicaçāo para Transferência de Tecnologia, Brasilia; EMBRAPA Florestas, Colombo, PR. pp. 15-18.

Figueiredo Ffilho, A., 2003. Etacional da Deposição de Serapilheira em uma Floresta Ombrófila Mista Localizada no Sul do Estado do Paraná. Ciência Florestal, Santa Maria, (13): 11-18.

FUNARBE. SAEG, 1993. Sistema para Análise Estatistica, v. 5.0, Viçosa, MG.

Gama-Rodrigues, A. C., 1997. Ciclagem de Nutrientes por Espécies Florestais em Povoamentos Puros e Mistos, em Solos de Tabuleiro da Bahia, Brasil. Tese (Doutorado em Solos e Nutrição de Plantas) Universidade Federal de Viçosa, Viçosa, 107 p.

Gama-Rodrigues, A. C., 2004. Ciclagem de Nutrientes em Sistemas Agroflorestais na Região Tropical: Funcionalidade e Sustentabilidade. in: Muller,'M. W., Gama-Rodrigues, A. C., Brandão, I. C. S. F. I. e Serôdio, M. H. de C. F. (eds.) Sistemas Agroflorestais, Tendência da Agricultura Ecológica nosTrópicos: Sustento da Vida e Sustento de Vida, Sociedade Brasileira de Sistemas Agroflorestais: Comissão Executiva do Plano de Lavoura Cacaueira, Ilhéus, BA; Campos dos Goytacazes, RJ. pp. 67-87.

Gama-Rodrigues, A. C.; Barros, N. F. e Mendonça, E. S., 1999. Alteraçōes Edáficas sob Plantios Puros e Mistos de Espécies Florestais Nativas do Sudeste da Bahia, Brasil. R. Bras. Ci. Solo, (23): 581-592.

Gama-Rodrigues, E. F. da, Gama-Rodrigues, A. C. da, Barros, N. F., 1997. Biomassa Microbiana de Carbono e de Nitrogênio de Solos sob Diferentes Coberturas Florestais. R. Bras. Ci. Solo, (21): 361-365.

Malavolta, E. A., 1980. Elementos de Nutrição Mineral de Plantas. São Paulo, CERES. 251p.

Monteiro, M. T., 2001. Carbono, Nitrogênio e Atividade da Biomassa Microbiana: Indicadores da Qualidade do Solo e da Serapilheira em Sitios Florestais do Norte Fluminense. Tese (Mestrado em'Produção Vegetal) - Universidade Estadual do Norte Fluminense, Campos dos Goytacazes, RJ, 77 p. 
Monteiro, M. T. e Gama-Rodrigues, E. F., 2004. Carbono, Nitrogênio e Atividade da Biomassa Microbiana em Diferentes Estruturas de Serapilheira de uma Floresta Natural. R. Bras. Ci. Solo, (28): 819-826.

Ndaw, S. M., 2003. Diversidade, Biomassa e Atividade Microbiana como Indicadores de Qualidade do Solo e da Serapilheira em Sitios sob Diferentes Coberturas Vegetais na Região Norte Fluminense. Tese (Mestrado em Produção Vegetal) - Universidade Estadual do Norte Fluminense, Campos dos Goytacazes, RJ, $98 \mathrm{p}$.

Paulino, G. M., 2003. Cobertura Florestal e Qualidade de Solo em Terras Degradadas no Norte Fluminense. Tese (Mestrado em Produçāo Vegetal) - Universidade Estadual do Norte Fluminense, Campos dos Goytacazes, RJ, 67p.

Rosolem, C. A., Calonego, J. C. e Foloni, J. S. S., 2003. Lixiviação de Potássio da Palha de Espécies de Cobertura de Solo de Acordo com a Quantidade de Chuva Aplicada. R. Bras. Ci. Solo, (27): 355-362.

van Soest, P. e Wine, R. H., 1968. Development of a Comphrehensive System of Feed Analysis and its Applications to Forages.J. Assoc. Official Agr. Chem., Madison, (51): 780-785.

Witschoreck, R., Schumacher, M. V., Caldeira, M. V. W., 2003. Estimativa da Biomassa e do Comprimento de Raizes Finas em Eucalyptus urophylla S.T. Blake no Municipio de Santa Maria - RS. Revista Arvore. 27 (2): $177-183$.

Zaia, F. C. e Gama-Rodrigues, A. C., 2004. Ciclagem de Nutrientes em Povoamentos de Eucalipto na Região Norte Fluminense, R. Bras. Ci. Solo, (28): 843-852. 


\section{REGLAMENTO DE PUBLICACION}

CIENCIA E INVESTIGACION FORESTAL es una publicación técnica, científica, arbitrada y seriada del Instituto Forestal de Chile, en la que se publica trabajos originales e inéditos, con resultados de investigaciones o avances de estas, realizados por sus propios investigadores y por profesionales del sector, del pais o del extranjero, que estén interesados en difundir sus experiencias en áreas relativas a las múltiples funciones de los bosques, en los aspectos económicos, sociales y ambientales. Consta de un volumen por año el que a partir del año 2007 está compuesto por tres números (abril, agosto y diciembre) y ocasionalmente números especiales.

La publicación cuenta con un Consejo Editor institucional que revisa en primera instancia los trabajos presentados y está facultado para aceptarlos, rechazarlos o solicitar modificaciones a los autores. Dispone además de un selecto grupo de profesionales externos y de diversos paises, de variadas especialidades, que conforma el Comité Editor. De acuerdo al tema de cada trabajo, estos son enviados por el Editor a al menos tres miembros del Comité Editor para su calificación especializada. Los autores no son informados sobre quienes arbitran los trabajos.

La revista consta de dos secciones; Artículos Técnicos y Apuntes, puede incluir además artículos de actualidad sectorial en temas seleccionados por el Consejo Editor o el Editor.

Artículos: Trabajos que contribuyen a ampliar el conocimiento científico o tecnológico, como resultado de investigaciones que han seguido un método científico.

Apuntes: Comentarios o análisis de temas particulares, que presenten enfoques metodológicos novedosos, representen avances de investigación, informen sobre reuniones técnicas o programas de trabajo y otras actividades de interés dentro del sector forestal o de disciplinas relacionadas. Los apuntes pueden ser también notas bibliográficas que informan sobre publicaciones recientes, en el pais o en el exterior, comentando su contenido e interés para el sector, en términos de desarrollo científico y tecnológico o como información básica para la planificación y toma de decisiones. 


\section{ESTRUCTURA DE LOS TRABAJOS}

\section{Artículos}

Los trabajos presentados para esta sección deberán contener Resumen, Summary, Introducción, Objetivos, Material y Método, Resultados, Discusión y Conclusiones, Reconocimientos (optativo) y Referencias. En casos muy justificados Apéndices y Anexos.

Título: El título del trabajo debe ser representativo del efectivo contenido del artículo y debe ser construido con el mínimo de palabras.

Resumen: Breve descripción de los objetivos, de la metodologia y de los principales resultados y conclusiones. Su extensión máxima es de una página y al final debe incluir al menos tres palabras clave que faciliten la clasificación bibliográfica del artículo. No debe incluir referencias, cuadros ni figuras. Bajo el título se identificará los autores y a pie de página su institución y dirección. El Summary es evidentemente la versión en inglés del Resumen.

Introducción: Como lo dice el título, este punto está destinado a introducir el tema, describir lo que se quiere resolver o aquello en que se necesita avanzar en materia de información, proporcionar antecedentes generales necesarios para el desarrollo o compresión del trabajo, revisar información bibliográfica y avances previos, situar el trabajo dentro de un programa más amplio si es el caso, y otros aspectos pertinentes. Los Antecedentes Generales y la Revisión de Bibliografía pueden en ciertos casos requerir especial atención y mayor extensión, si así fuese, en forma excepcional puede ser reducida la Introducción a lo esencial e incluir estos puntos separadamente.

Objetivos: Breve enunciado de los fines generales del artículo o de la línea de investigación a que corresponda y definición de los objetivos especificos del artículo en particular.

Material y Método: Descripción clara de la metodologia aplicada y, cuando corresponda, de los materiales empleados en las investigaciones o estudios que dan origen al trabajo. Si la metodología no es original se deberá citar claramente la fuente de información. Este punto puede incluir Cuadros y Figuras, siempre y cuando su información no resulte repetida con la entregada en texto. 
Resultados: Punto reservado para todos los resultados obtenidos, estadisticamente respaldados cuando corresponda, y asociados directamente a los objetivos especificos antes enunciados. Puede incluir Cuadros y Figuras indispensables para la presentación de los resultados o para facilitar su comprensión, igual requisito deben cumplir los comentarios que aqui se pueda incluir.

Discusión y Conclusiones: Análisis e interpretación de los resultados obtenidos, sus limitaciones y su posible trascendencia. Relación con la bibliografía revisada y citada. Las conclusiones destacan lo más valioso de los resultados y pueden plantear necesidades consecuentes de mayor investigación o estudio o la continuación lógica de la línea de trabajo.

Reconocimientos: Punto optativo, donde el autor si lo considera necesario puede dar los créditos correspondientes a instituciones o personas que han colaborado en el desarrollo del trabajo o en su financiamiento. Obviamente se trata de un punto de muy reducida extensión.

Referencias: Identificación de todas las fuentes citadas en el documento, no debe incluir referencias que no han sido citadas en texto y deben aparecer todas aquellas citadas en éste.

Apéndices y Anexos: Deben ser incluidos sólo si son indispensables para la comprensión del trabajo y su incorporación se justifica para reducir el texto. Es preciso recordar que los Apéndices contienen información o trabajo original del autor, en tanto que los Anexos contienen información complementaria que no es de elaboración propia.

\section{Apuntes}

Los trabajos presentados para esta sección tienen en principio la misma estructura descrita para los artículos, pero en este caso, según el tema, grado de avance de la investigación o actividad que los motiva, se puede adoptar una estructura más simple, obviando los puntos que resulten innecesarios.

\section{PRESENTACION DE LOS TRABAJOS}

La Revista acepta trabajos en español y ocasionalmente en inglés o portugués, redactadas en lenguaje universal, que pueda ser entendido no sólo por especialistas, de modo de cumplir su objetivo de transferencia de conocimientos y difusión al sector forestal en general. No se acepta redacción en primera persona. 
Formato tamaño carta $(21,6 \times 27,9 \mathrm{~cm})$, márgenes $2,5 \mathrm{~cm}$ en todas direcciones, espacio simple y un espacio libre entre párrafos. Letra Arial 10. Un tab (8 espacios) al inicio de cada párrafo. No numerar páginas. Extensión máxima trabajos 25 carillas para artículos y 15 para Apuntes. Justificación ambos lados.

Primera página incluye título en mayúsculas, negrita, centrado, letra Arial 12, una línea, eventualmente dos como máximo. Dos espacios bajo éste: Autor (es), minúsculas, letra 10 y llamado a pie de página indicando Institución, pais y correo electrónico en letra Arial 8. Dos espacios más abajo el Resumen y, si el espacio resulta suficiente, el Summary. Si no lo es, página siguiente igual que anterior, el Summary.

En el caso de los Apuntes, en su primera página arriba tendrán el título del trabajo en mayúscula, negrita, letra 12 y autor (es), institución, pais y correo, letra 10 , normal minúsculas, bajo una línea horizontal, justificado a ambos lados, y bajo esto otra linea horizontal. Ej:

\section{EL MANEJO FORESTAL SOSTENIBLE COMO MOTOR DE EMPRENDIMIENTO DEL MUNDO RURAL: LA EXPERIENCIA EN} CHILE._Victor Vargas Rojas. Instituto Forestal. Ingeniero Forestal. Mg. Economia de Recursos Naturales y del Medio Ambiente.vvargas@infor.cl

Titulo puntos principales (Resumen, Summary, Introducción, Objetivos, etc) en mayúsculas, negrita, letra 10, margen izquierdo. Sólo para Introducción usar página nueva, resto puntos principales seguidos, separando con un espacio antes y después de cada uno. Títulos secundarios en negrita, minúsculas, margen izquierdo. Titulos de tercer orden minúsculas margen izquierdo. Si fuesen necesarios títulos de cuarto orden, usar minúsculas, un tab (7 espacios) y anteponer un guión y un espacio. Entre sub títulos y párrafos precedente y siguiente un espacio libre. En sub titulos con más de una palabra usar primera letra de palabras principales en mayúscula. No numerar puntos principales ni sub títulos.

Nombres de especies vegetales o animales: Vulgar o vernáculo en minúsculas toda la palabra, seguido de nombre en latín o cientifico entre paréntesis la primera vez que es mencionada la especie en el texto, en cursiva (no negrita), minúsculas y primera letra del género en mayúsculas. Ej. pino o pino radiata (Pinus radiata).

Citas de referencias bibliográficas: Sistema Autor, año. Ejemplo en citas en texto; De acuerdo a Rodriguez (1995) el comportamiento de...., o el comportamiento de... (Rodriguez, 1995). Si son dos autores; De acuerdo a Prado y Barros (1990) el comportamiento de ..., o el comportamiento de ... (Prado y Barros, 1990). Si son 
más de dos autores; De acuerdo a Mendoza et al. (1990), o el comportamiento ... (Mendoza et al., 1990).

En el punto Referencias deben aparecer en orden alfabético por la inicial del apellido del primer autor, letra 8, todas las referencia citadas en texto y sólo estas. En este punto la identificación de la referencia debe ser completa: Autor (es), año. En negrita, minúsculas, primeras letras de palabras en mayúsculas y todos los autores en el orden que aparecen en la publicación, aquí no se usa et al. A continuación, en minúscula y letra 8 , primeras letras de palabras principales en mayúscula, título completo y exacto de la publicación, incluyendo institución, editorial y otras informaciones cuando corresponda. Margen izquierdo con justificación ambos lados. Ejemplo:

En texto: (Yudelevich et al., 1967) o Yudelevich et al. (1967) señalaron ...

\section{En referencias:}

Yudelevich, Moisés; Brown, Charles y Elgueta, Hernán, 1967. Clasificación Preliminar del Bosque Nativo de Chile. Instituto Forestal. Informe Técnico $N^{\circ} 27$. Santiago, Chile.

Expresiones en Latín, como et al.; a priori y otras, asi como palabras en otros idiomas como stock, marketing, cluster, stakeholders, commodity y otras, que son de frecuente uso, deben ser escritas en letra cursiva.

Cuadros y Figuras: Numeración correlativa: No deben repetir información dada en texto. Sólo se aceptan cuadros y figuras, no así tablas, gráficos, fotos u otras denominaciones. Toda forma tabulada de mostrar información se presentará como cuadro y al hacer mención en texto (Cuadro $N^{\circ} 1$ ). Gráficos, fotos y similares serán presentadas como figuras y al ser mencionadas en texto (Figura $N^{0} 1$ ). En ambos casos aparecerán enmarcados en línea simple y centrados en la página. En lo posible su contenido escrito, si lo hay, debe ser equivalente a la letra Arial 10 u 8 y el tamaño del cuadro o figura proporcionado al tamaño de la página. Cuadros deben ser titulados como Cuadro $\mathrm{N}^{\circ}$, minúsculas, letra 8 , negrita centrado en la parte superior de estos, debajo en mayúsculas, negritas letra 8 y centrado el título (una linea en lo posible). Las figuras en tanto serán tituladas como Figura $\mathrm{N}^{\circ}$, minúscula, letra 8 , negrita, centrado, en la parte inferior de estas, y debajo en mayúsculas, letra 8 , negrita, centrado, el título (una línea en lo posible). Si la diagramación y espacios lo requieren es posible recurrir a letra Arial narrow. Cuando la información proporcionada por estos medios no es original, bajo el marco debe aparecer entre paréntesis y letra 8 la fuente o cita que aparecerá también en referencias. Si hay simbolos u otros elementos que requieren explicación, se puede proceder de igual forma que con la fuente. 
Se aceptan fotos en blanco y negro y en colores, siempre que reúnan las caracteristicas de calidad y resolución que permitan su impresión.

Abreviaturas, magnitudes y unidades deben estar atenidas a la Norma NCh 30 del Instituto Nacional de Normalización (INN). Se empleará en todo caso el sistema métrico decimal. Al respecto es conveniente recordar que la unidades se abrevian en minúsculas, sin punto, con la excepción de litro (L) y de aquellas que provienen de apellidos de personas como grados Celsius $\left({ }^{\circ} \mathrm{C}\right)$. Algunas unidades de uso muy

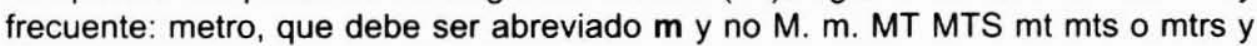
otras formas como a menudo se ve en las carreteras y otros lugares; metro cúbico $\mathrm{m}^{3}$, metro ruma $\mathrm{mr}$; o hectáreas ha y no HTA HAS há o hás.

Llamados a pie de página: Cuando estos son necesarios, serán numerados en forma correlativa para cada página, no de 1 a n a lo largo del trabajo. Aparecerán al pie en letra 8 . No usar este recurso para citas bibliográficas, que deben aparecer como se indica en Referencias.

Archivos protegidos, "sólo lectura" o PDF serán rechazados de inmediato porque no es posible editarlos. La Revista se reserva el derecho de efectuar todas las modificaciones de carácter formal que el Comité Editor o el Editor estimen necesarias o convenientes, sin consulta al autor. Modificaciones en el contenido evidentemente son consultadas por el Editor al autor, si no hay acuerdo se recurre nuevamente al Consejo Editor o los miembros de este que han participado en el arbitraje o calificación del trabajo.

\section{ENVIO DE TRABAJOS}

Procedimiento electrónico. En general bastará enviar archivo Word, abierto al Editor sbarros@infor.gob.cl

Cuadros y figuras ubicadas en su lugar en el texto, no en forma separada. EI Editor podrá en algunos casos solicitar al autor algún material complementario en lo referente a cuadros y figuras (archivos Excel, imágenes, figuras, fotos, por ejemplo).

El autor deberá indicar si propone el trabajo para Articulo o Apunte y asegurarse de recibir confirmación de la recepción conforme del trabajo por parte del Editor.

Respecto del peso de los archivos, tener presente que $1 \mathrm{Mb}$ es normalmente el limite razonable para los adjuntos por correo electrónico. No olvidar que las imágenes son pesadas, por lo que siempre al ser pegadas en texto Word es 
conveniente recurrir al pegado de imágenes como JPEG o de planillas Excel como Metarchivo Mejorado.

En un plazo de 30 dias desde la recepción de un trabajo el Editor informará al autor principal sobre su aceptación (o rechazo) en primera instancia e indicará (condicionado al arbitraje del Comité Editor) el Volumen y Número en que el trabajo seria incluido. Posteriormente enviará a Comité Editor y en un plazo no mayor a 3 meses estará sancionada la situación del trabajo propuesto. Si se mantiene la información dada por el Editor originalmente, el trabajo es aceptado como fue propuesto (Articulo o Apunte) y no hay observaciones de fondo, el trabajo es editado y pasa a publicación cuando y como se informó al inicio. Si no es asi, el autor principal será informado sobre cualquier objeción, observación o variación, en un plazo total no superior a 4 meses. 


\section{CIENCIA E INVESTIGACION FORESTAL}

\section{ARTICULOS}

PAGINA

EL GENERO ACACIA, ESPECIES MULTIPROPOSITO. Santiago Barros. Chile.

PROCESOS INDUSTRIALES Y APLICACIONES DE ACACIA EN CHILE. Rodrigo Briones y Juan Carlos Pinilla. Chile.

RESENA Y FUTURO DEL SECTOR FORESTAL REGIÓN DE COQUIMBO. CHILE. Juan Cerda. Chile.

EFFECT OF ACACIA MELANOXYLON WOOD DENSITY ON PAPERMAKING POTENTIAL CarIa Delgado, Antonio Santos, Rogerio Simoes and Ofelia Anjos. Portugal.

EFFECTS OF ACACIA PLANTATION ON TREE SPECIES COMPOSITION, SOIL PROPERTIES MINERALIZATION AND MICROCLIMATE IN GRASSLAND OF MT. MAKILING. PHILIPPINES. Yong Kwon Lee, Don Koo Lee, Su Young Woo and Pil Sun Park. Korea.

IMPROVED PROSPECTS FOR THE DOMESTICATION OF ACACIA SALIGNA IN REGION DE COQUIMBO, CHILE. Maurice W. McDonald, Richard Mazanec, John R. Bartle and Bruce R. Maslin. Australia.

TROPICAL ACACIAS: THEIR DOMESTICATION AND CONRIBUTION TO ASIA S WOOD AND PULP INDUSTRIES. Stephen Midgley. Australia.

AVANCES EN LA ETRATEGIA DE MEJORAMIENTO GENÉTICO PARA ESPECIES DEL GENERO ACACIA EN LA ZONA CENTRO SUR DE CHILE. Maria Paz Molina y Juan Carlos Pinilla S. Chile.

ESTIMACION DE COMPONENTES DE VARIANZA Y PREDICCION DE VALORES GENETICOS EN POBLACIONES DE ACACIA AZUL USANDO EL ALGORITMO DE CADENAS INDEPENDIENTES Freddy Mora, Sandra Perret, Carlos Alberto Scapim, Elias Martins y Maria Paz Molina. Chile.

MICROPROPAGACIÓN DE ÁRBOLES SUPERIORES DE ACACIA MELANOXYLONR. BR. Oriana Ortiz, Maria Elisa González y Laura Koch. Chile.

OPCIONES PRODUCTIVAS CON ACACIAS PARA CHILE. Juan Carlos Pinilla S., Maria Paz Molina B. y Braulio Gutièrrez C. Chile.

PLANTACIONES DE ACACIA UNA OPCION DE GESTION FORESTAL PARA PROPIETARIOS FORESTALES. Juan Carlos Pinilla S., Maria Paz Molina B. y Marta González. Chile.

ESPECIES FORESTALES CON DIVERSIDAD DE USOS EN UN BOSQUE TROPICAL CADUCIFOLIO DE LA COMUNIDAD INDIGENA DE TOMATLAN, JALISCO, MÉXICO. Maria Leonor Román Miranda, Antonio Mora Santacruz, Servando Carvajal Hernández y Héctor Ochoa Ruiz. México.

ACÜMULO E CARACTERIZAÇO DA SERAPILHEIRA E FERTILIDADE DO SOLO EM COBERTURAS FLORESTAIS NO NORTE FLUMINENSE. Miriam Peixoto Soares da Silva, Antonio Carlos da GamaRodrigues e Deborah Guerra Barroso. Brasil.
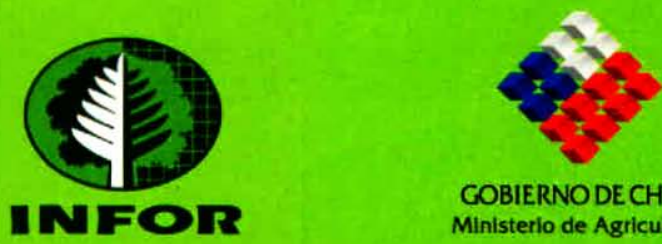